\title{
Über Fernsprech-Unterwasserkabel.
}

\author{
Von F. Lïschen.
}

(Mitteilung aus dem Kaiserlichen Telegraphenversuchsamt.)

Einleitung. Als Isoliermaterial ist bisher für Fernsprech-Unterwasserkabel Guttapercha oder Papier verwendet worden. Z. B. sind die im Jahre Igo3 verlegten Kabel Fehmarn-Lolland (I9,3 km), Greetsiel-Borkum $(29,5 \mathrm{~km})$ und Kuxhaven-Helgoland $(75,2 \mathrm{~km})$ mit Papier isoliert, und zwar ist in dem erstgenannten das Papier imprägniert und fest um den Leiter gelegt, während in den letztgenannten zur Herabminderung der Kapazität die Leiter mit Papier hohl umsponnen sind. Alle drei Kabel haben nach K ra rup zur Erhöhung der Induktivität mit Eisendraht umsponnene Leiter erhalten. Auch das Pupinkabel durch den Bodensee ist ein Papierkabel. Demgegenüber sind z. B. die Kabel Helsingör-Helsingborg sowie alle zwischen England und dem Festlande verlaufenden Fernsprechkabel mit Guttapercha isoliert. Das im Jahre Igro zwischen England und Frankreich sowie das letzte im Jahre IgII zwischen England und Belgien verlegte Fernsprechkabel sind Pupinkabel, das zwischen England und Frankreich in diesem Jahre ausgelegte Kabel hat eine durch Eisenbespinnung erhöhte Induktivität erhalten.

Welches Isoliermaterial im Einzelfalle zu wählen ist, muß nach den mechanischen und elektrischen Eigenschaften sowie nach den Kosten der verschiedenen Kabelmuster entschieden werden. Das gleiche gilt für die Wahl des Mittels zur Erhöhung der Induktivität. .

1. Mechanische Eigenschaften der Papier-Luftraum- und Guttaperchakabel. Bezüglich der mechanischen Eigenschaften sind die Guttaperchakabel den Papier-Luftraumkabeln in mehrfacher Beziehung überlegen. Es sei hier nur kurz auf folgende Vorzüge der Guttaperchakabel hingewiesen. Sie widerstehen dem Wasserdruck in größeren Tiefen besser, sie sind weniger Beschädigungen durch Strömungen und Schiffahrt ausgesetzt und sie sind leichter zu verlegen.

Die vorher erwähnten Papierkabel sind alle in verhältnismäßig flachen Gewässern verlegt worden, deren Tiefe $40 \mathrm{~m}$ nicht übersteigt. Eine Ausnahme macht nur das Pupinkabel durch den Bodensee, das an seiner tiefsten Stelle einem Wasserdruck von $250 \mathrm{~m}$ Höhe (etwa $25 \mathrm{Atm}$.) ausgesetzt ist. In solchen Tiefen können gewöhnliche Papierkabel mitBleimantel nicht mehr verlegt werden. Bei normalem, bis $5 \mathrm{~mm}$ Wandstärke reichendem Bleimantel halten die Kabel dem Wasserdruck nicht stand. Bei Versuchen mit 50 Atm. Druck, die von der Firma Siemens \& Halske anläßlich der Verlegung des Bodenseekabels angestellt worden sind, wurden sämtliche Kabelmuster mit Mänteln aus reinem oder zinnlegiertem Blei aus der Form gebracht. Schließlich gelang es zwar durch Umspinnung der Seele mit einem $2 \mathrm{~mm}$ starken Stahldraht, über den dann der Bleimantel aufgebracht wurde, dem Kabel genügende Festigkeit zu geben. Immerhin bleibt diese Notwendigkeit gegenüber den Guttaperchakabeln eine lästige Beigabe ${ }^{1}$ ).

Auch bei gleichstarker Bewehrung werden Papierkabel leichter beschädigt als Guttaperchakabel. Beim Hin- und Herzerren durch Strömungen, Flut und Ebbe, durch schleifende Anker oder Fischnetze kann unter Umständen der Bleimantel beschädigt werden, selbst wenn die Bewehrung den Angriffen standhält. Besonders nachteilig ist dabei für die Papierkabel, daß bei einer Beschädigung des Bleimantels stets ein mehr oder weniger großer Teil des Kabels wegen der hygroskopischen Eigenschaften des Papiers unbrauchbar wird, wodurch sich die Wiederherstellung schwieriger, zeitraubender und kostspieliger gestaltet.

1) Vgl. Ebeling, Über das im Bodensee verlegte Fernsprechkabel mit Selbstinduktionsspulen nach dem Pupinschen System. ETZ. I907, S. 66r. 
Ein anderen Nachteil der Papierkabel ist der, daß sie durch den Bleimantel außergewöhnlich schwer werden. Bei größeren Tiefen würden die heutigen Kabeischiffe schon aus diesem Grunde Papierkabel mit Bleimantel gar nicht ohne kostspielige Umänderung ihrer Einrichtungen verlegen können.

Aus diesen hier nur kurz angedeuteten Gründen kommen daher Papierkabel nur an flachen Stellen in Betracht, wo sie weder starken Strömungen noch Beschädigungen durch schleifende Anker usw. ausgesetzt sind.

2. Elektrische Eigenschaften der Papier-Luftraumkabel und der Guttaperchakabel. Für die Beurteilung der elektrischen Güte eines Isoliermittels sind zwei Größen zu betrachten: die Kapazität und die Ableitung. An Stelle der Ableitung gibt man im allgemeinen das Verhältnis Ableitung $(G)$ : Kapazität $(C)$ bei einer Kreisfrequenz von $\omega=5000$, der mittleren Frequenz der Fernsprechströme, an. Es wäre vielleicht praktischer, für die Charakterisierung des Isoliermaterials das Verhältnis $\frac{\mathrm{G}}{{ }_{\omega} \mathrm{C}}$ zu wählen, da die Ableitung der Frequenz der Wechselströme, wenigstens für den Bereich der Fernsprechströme, angenähert proportional ist, also $\frac{G}{\omega C}$ eine von der Frequenz unabhängige Materialkonstante ist. Den Wert für $\mathrm{G} / \mathrm{C}$ anzugeben, hat sich aber namentlich in England und Amerika so eingebürgert, daß es nicht zweckmäßig erscheint, von dieser Übung abzugehen.

Je kleiner das Verhältnis G/C ist, desto besser ist das Material. Der Wert schwankt für verschiedene Sorten gleichen Materials erheblich. So beträgt er für die bisher übliche Guttaperchamischung 80 bis über IOO, z. B. in dem neuen französisch-englischen Fernsprechkabel für eine Doppelleitung Iog, für die zweite 87,5 ${ }^{1}$ ), während er für das neue englisch-belgische Kabel nach den Messungen der Firma Siemens Brothers, London, nur I2 beträgt. Das von Siemens erreichte Ergebnis ist danach als ein großer Erfolg anzusprechen. Mit der für das englisch-belgische Kabel verwendeten Guttapercha kann man z. B. in einer Pupinleitung, deren Ohmscher Widerstand für das Kilometer $\mathrm{I}_{4}, \mathrm{O} \mathrm{Ohm}$ und deren Kapazität $0, I 8 \mu \mathrm{F}$ beträgt, und in die Spulen mit einer wirksamen Zeitkonstante von 0,02 eingeschaltet werden, eine spezifische Dämpfung von 0,0125 erreichen, mit einer Guttapercha, bei der das Verhältnis $\mathrm{G} / \mathrm{C}=$ Ioo ist, aber nur eine solche von 0,0I95. Das letztgenannte Kabel würde also um $56 \%$ schlechter sein.

Auch für Papier-Luftraumkabel schwankt der Wert von $\mathrm{G} / \mathrm{C}$ erheblich; er liegt.etwa zwischen I5 und roo, je nach der Papiersorte, der Konstruktion und dem Grade der Trocknung.

Will man zwei Materialien mit verschiedener Dielektrizitätskonstante vergleichen, so genügt es nicht, den Wert G/C für sich zu betrachten, man mußvielmehr auch die absoluten Werte der Kapazität berücksichtigen. Am übersichtlichsten erscheint es, die Dämpfung gleichdimensionierter Kabel zu untersuchen, um ein vergleichendes Urteil über die elektrische Güte zweier Isoliermittel zu gewinnen.

Die Fortpflanzungskonstante einer Fernsprech-Doppelleitung, deren reeller Teil den Wert der spezifischen Dämpfung gibt, ist

$$
\gamma=\sqrt{(\mathrm{R}+\mathrm{i} \omega \mathrm{L})(\mathrm{G}+\mathrm{i} \omega \mathrm{C})}
$$

wo R, L, G und C die kilometrischen Werte des Widerstandes, der Induktivität, der Ableitung und der Kapazität, $\omega$ die Kreisfrequenz und i die $\sqrt{-x}$ bedeutet. Führt man das Verhältnis $\mathrm{G} / \mathrm{C}=$ a ein, und setzt die Kapazität gleich dem Produkt aus der Dielektrizitätskonstanten und einer nur von den Dimensionen abhängigen Konstanten D:

$$
\mathrm{C}=\varepsilon \mathrm{D},
$$

1) Annales des Postes, des Télégraphes et des Téléphones I912, S. 543. G $=1,032 \cdot 10^{-5}$ und $0,83 \cdot 10^{-5}$ Siemens, $C=0,0946 \cdot 10^{-6}$ und $0,095 \cdot 10^{-6}$ Farad. 
so ergibt sich

$$
\gamma=\sqrt{(\mathrm{R}+\mathrm{i} \omega \mathrm{L})(\mathrm{a}+\mathrm{i} \omega) \varepsilon \mathrm{D} .}
$$

Sieht man davon ab, daß bei gleichdimensioniertem Leiter in den Kabeln mit Bleimantel der wirksame Widerstand wegen der Verluste durch Induktionsströme im Bleimantel höher als in Kabeln ohne Bleimantel ist, so verhalten sich die Fortpflanzungskonstanten zweier gleichdimensionierter Kabel, deren Isoliermittel durch die Größen $\mathrm{a}_{1}$ und $\varepsilon_{1}$ sowie $\mathrm{a}_{2}$ und $\varepsilon_{2}$ charakterisiert sind, wie

$$
\gamma_{1}: \gamma_{2}=\sqrt{\left(a_{1}+i \omega\right) \varepsilon_{1}}: \sqrt{\left(a_{2}+i \omega\right) \varepsilon_{2}} .
$$

Die spezifischen Dämpfungen verhalten sich wie die reellen Teile der Wurzeln.

In einer Pupinleitung, die so pupinisiert ist, daß die Dämpfung ein Minimum ist, beträgt die spezifische Dämpfung, wenn man die natürliche Induktivität der Leitung nicht berücksichtigt und mit $\tau$ die Zeitkonstante der Spulen bezeichnet,

$$
\beta=\sqrt{R\left(G+\frac{C}{\tau}\right)} .
$$

Unter Berücksichtigung der Induktivität der Leitung ergibt sich

$$
\beta=\sqrt{\left(R-\frac{L}{\tau}\right)\left(G+\frac{C}{\tau}\right)}=\sqrt{\left(R-\frac{L}{\tau}\right)\left(a+\frac{I}{\tau}\right) \varepsilon D .}
$$

Die spezifischen Dämpfungen bei Verwendung verschiedener Isoliermittel verhalten sich also:

$$
\beta_{1}: \beta_{2}=\sqrt{\left(a_{1}+\frac{I}{\tau}\right) \varepsilon_{1}}: \sqrt{\left(a_{2}+\frac{I}{\tau}\right) \varepsilon_{2}} .
$$

Nach diesen Formeln ist berechnet worden, wie sich die spezifischen Dämpfungen in gleichdimensionierten Papier-Luftraumleitern und Guttaperchaleitern verhalten. Es ist dabei für die Zeitkonstante der Spulen ein Wert von o,03, d. h. ein günstiger aber ohne Schwierigkeiten erreichbarer Wert, angenommen worden. Die Dielektrizitätskonstante der Papier-Luftraumkabel ist mit einem Wert von 1,6 und die der Guttapercha mit einem Wert von 3,4 angesetzt worden.

Es ergibt sich dann folgendes:

Papier-Luftraum-Kabel.

Guttaperchakabel.

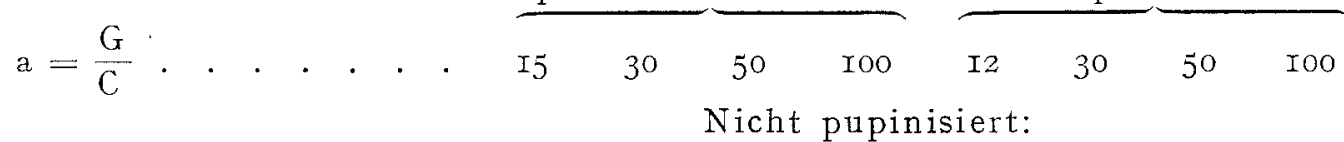

Verhältnis der spezifischen

Dämpfungen . . . . I,000 : I,00I : I,003:I,008 : I,457:I,460:I,463:I,470

Pupinisiert:

dgl. I,000 : I,I45:I,3I3:I,66I : I,4I3 : I,669:I,9I4: 2,42I

Aus der Zusammenstellung ersieht man zunächst die bekannte Tatsache, daß die Ableitung in -nicht pupinisierten Kabeln keine wesentliche Bedeutung hat. Wenn bei gleicher Dielektrizitätskonstante das Verhältnis G/C von I5 auf Ioo wächst, so beträgt der Unterschied in der Dämpfung noch nicht I v. H. Demgegenüber beträgt in Pupinkabeln der Dämpfungszuwachs in diesem Falle 66 v. H.

Für den Vergleich zwischen Papier-Luftraumkabeln und Guttaperchakabeln ist aus der Zusammenstellung zu entnehmen, daß bei gleichen Dimensionen in dem mit der besten bisher hergestellten Guttapercha isolierten Kabel die Dämpfung immer noch um reichlich 40 v. H. größer ist als in dem besten Papier-Luftraumkabel. Auch wenn man berücksichtigt, daß in starkdrähtigen Papier-Luftraumkabeln durch die Verluste im Bleimantel die Dämpfung möglicherweise noch um mehrere Prozent erhöht wird, ändert sich dies Verhältnis 
nicht wesentlich. Die Verbesserung der Papier-Luftraumkabel hat eben gleichen Schritt mit der der Guttaperchakabel gehalten.

Wo daher nicht die mechanischen Anforderungen Papierkabel ausschließen, sind diese ihrer elektrischen Eigenschaften wegen, trotz der erhebliehen Verbesserung der Guttapercha, den Guttaperchakabeln vorzuziehen.

Ob man Krarup- oder Pupinkabel verwenden will, ist in erster Linie eine Frage der Kosten. Man kann nicht sagen, daß man mit einem Krarupkabel nicht eine so geringe Dämpfung erreichen könne wie mit einem Pupinkabel. Es fragt sich nur, mit welchem Materialaufwand und welchen Kosten es erreicht wird. Einen interesssanten Vergleich bieten da die beiden neuesten Unterwasserkabel durch den Kanal, von England nach Belgien uṇ nach Frankreich. Das erste ist ein Pupinkabel, das zweite ein Krarupkabel. Die spezifische Dämpfung beider Kabel ist annähernd gleich, sie beträgt für das Pupinkabel 0,00980 für das Krarupkabel 0,00985 bei einer Kreisfrequenz der Wechselströme von $\omega=5000$. Dabei beträgt das Gewicht des Kupfers für das Kilometer im Pupinkabel $45 \mathrm{~kg}$, in dem Krarupkabel $84,5 \mathrm{~kg}$, also annähernd doppelt soviel. Das Guttaperchagewicht ist in beiden Kabeln das gleiche, nämlich 42,5 kg für das Kilometer. In dem Pupinkabel besteht der Leiter aus einer einfach herzustellenden siebendrähtigen Litze, in dem Krarupkabel aus einem mittleren Draht, der mit sechs Fassondrähten umgeben ist. Diese Bauart war notwendig, da sich um eine Litze der Eisendraht schlecht umwickeln läßt. Preise sind in den Veröffentlichungen nicht angegeben; es liegt aber nach Vorstehendem auf der Hand, daß selbst bei hohem Preise der Spulen in einem Pupinkabel mit geringeren Kosten eine bestimmte Dämpfung zu erreichen ist als in einem Krarupkabel.

In elektrischer Beziehung sind Pupinkabel insofern den Krarupkabeln im allgemeinen vorzuziehen, als sie eine höhere Charakteristik haben. Z. B. hat das Krarupkabel zwischen England und Frankreich eine Charakteristik von $3=278 \cdot \epsilon^{-\mathbf{i} 2,5^{\circ}}$. Für das Pupinkabel zwischen England und Belgien wird eine Charakteristik von etwa 800 angegeben. Es ist demnach seiner Charakteristik nach sowohl zur Einschaltung in Pupinleitungen wie auch in gewöhnliche Freileitungen geeignet. Schaltet man dagegen das Krarupkabel in Pupinleitungen ein, so entstehen erhebliche Verluste durch Reflexionen, es ist also immerhin nur beschränkt verwendbar und gerade in Verbindungen auf weite Entfernungen, für die heute nur noch Pupinfreileitungen in Frage kommen, weniger geeignet. Bei der Einschaltung von $\left.43^{1}\right) \mathrm{km}$ Krarupkabel des obigen Musters zwischen zwei $500 \mathrm{~km}$ lange $4,5 \mathrm{~mm}$ starke Pupinleitungen mit einer Charakteristik von rund I950 ergibt sich eine Erhöhung des Dämpfungsexponenten durch Reflexionen um 0,88 . Da der Dämpfungsexponent des Kabels für sich nur 0,42 beträgt, übersteigt also der Dämpfungszuwachs durch Reflexionen die eigentliche Dämpfung um mehr als $200 \%$. Nun kann man zwar durch Einschaltung von Übertragern diesen Betrag herabdrücken. Dann hat man aber immer noch die Verluste in den Ubertragern. Bei den besten bisher im Gebrauch befindlichen Fernsprechübertragern beträgt der Wirkungøgrad etwa $85 \%$. Da man zwei Übertrager einschalten muß - an beiden Enden des Kabels - muß man also immer noch mit einer Vergrößerung des Dämpfungsexponenten um $2 \times 0,15=0,3$ rechnen. Das bedeutet für den vorliegenden Fall immer noch eine Erhöhung der Dämpfung des Kabels um $70 \%$

Da die Berechnungen über den Dämpfungszuwachs bei Aneinanderschaltung mehrerer Leitungen bisher wenig üblich sind, so dürfte es manchem erwünscht sein, wenn bei dieser Gelegenheit der Gang einer solchen Rechnung mit einigen praktischen Hinweisen zahlenmäßig an einem Beispiel durchgeführt wird.

3. Berechnung von Reflexionsverlusten. Es soll der Dämpfungszuwachs berechnet werden, der durch. Reflexionen entsteht,

1) Das ist die tatsächliche Länge des Kabels. 
a) für den Fall, daß an ein Krarupkabel von $43 \mathrm{~km}$ Länge der Bauart des englischfranzösischen Kabels eine oberirdische Pupinleitung von $500 \mathrm{~km}$ Länge angeschaltet wird,

b) für den Fall, daß ein solches Kabel zwischen zwei oberirdische Pupinleitungen von je $500 \mathrm{~km}$ Länge eingeschaltet wird.

a) Es seien $\mathfrak{U}^{\prime} \mathfrak{B}^{\prime} \mathfrak{C}^{\prime}$ (s. Fig. I) die Konstanten des Kabels, $\mathfrak{Q}^{\prime \prime} \mathfrak{B}^{\prime \prime} \mathfrak{C}^{\prime \prime}$ die Konstanten der Pupinleitung, $\mathfrak{F}_{\mathfrak{a}}$, $\widetilde{\mathfrak{J}}_{\mathfrak{a}}$ Spannung und Strom am Anfang des Kabels, $\mathfrak{B}$, $\mathfrak{\Im}$ Spannung und Strom am Ende des Kabels oder Anfang der Pupinleitung und $\mathfrak{F}_{\mathrm{e}}$, $\mathfrak{\Im}_{\mathrm{e}}$ Spannung und Strom am Ende der Pupinleitung, $\mathfrak{A}_{1} \mathfrak{A}_{2} \mathfrak{S} \mathfrak{C}$ die Konstanten des ganzen Systems. Dann bestehen folgende Beziehungen:

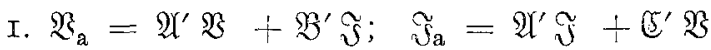

2. $\mathfrak{\mathfrak { V }}=\mathfrak{A}^{\prime \prime} \mathfrak{B}_{\mathrm{e}}+\mathfrak{B}^{\prime \prime} \mathfrak{V}_{\mathrm{e}} ; \mathfrak{\mathfrak { I }}=\mathfrak{A}^{\prime \prime} \widetilde{\mathfrak{V}}_{\mathrm{e}}+\mathfrak{C}^{\prime \prime} \mathfrak{B}_{\mathrm{e}}$

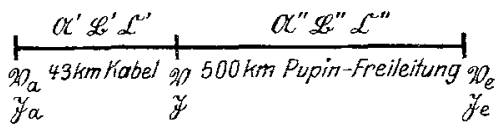

Fig. I.

3. $\mathfrak{V}_{\mathrm{a}}=\mathfrak{A}_{1} \mathfrak{B}_{\mathrm{e}}+\mathfrak{B} \mathfrak{\mathfrak { J }}_{\mathrm{e}} ; \quad \mathfrak{J}_{\mathrm{a}}=\mathfrak{U}_{2} \mathfrak{\mathfrak { V }}_{\mathrm{e}}+\mathfrak{C} \mathfrak{V}_{\mathrm{e}}$.

Durch Elimination von $\mathfrak{F}$ und $\mathfrak{\Im}$ aus I. und 2. ergeben sich die Konstanten des ganzen Systems folgendermaßen:

$$
\begin{aligned}
& \mathfrak{U}_{1}=\mathfrak{U}^{\prime} \mathfrak{U}^{\prime \prime}+\mathfrak{B}^{\prime} \mathfrak{C}^{\prime \prime} \\
& \mathfrak{U}_{2}=\mathfrak{U}^{\prime} \mathfrak{U}^{\prime \prime}+\mathfrak{C}^{\prime} \mathfrak{B}^{\prime \prime} \\
& \mathfrak{Z}^{2}=\mathfrak{U}^{\prime} \mathfrak{S}^{\prime \prime}+\mathfrak{B}^{\prime} \mathfrak{A}^{\prime \prime} \\
& \mathfrak{C}=\mathfrak{U}^{\prime} \mathfrak{C}^{\prime \prime}+\mathfrak{C}^{\prime} \mathfrak{Q}^{\prime \prime}
\end{aligned}
$$

Den Dämpfungszuwachs durch Reflexionen erhält man, indem man aus den Konstanten $\mathfrak{U}_{1}, \mathfrak{A}_{2}, \mathfrak{B}$, $\mathfrak{C}$ den Dämpfungsexponenten des ganzen Systems berechnet und davon die algebraische Summe der Dämpfungsexponenten der Teile abzieht.

Zunächst sind also die Konstanten der beiden Leitungen zu berechnen.

I. Kabel

\section{Angegeben wird}

die Charakteristik mit . . . . $3=278,5 \cdot \mathrm{e}^{-\mathrm{i} 2^{0} 17^{\prime}}$

die Fortpflanzungskonstante . . $\gamma=\beta+i \alpha=0,00985+i 0,1655$

bei $43 \mathrm{~km}$ Länge ergibt sich . . $\gamma \cdot \gamma=\beta 1+i \alpha 1=0,423+i$ 7,I 6 .

Die Wellenlängenkonstante $\alpha$ l beträgt also mehr als $2 \pi$, d. h. die Ströme (und Spannungen) am Anfang und am Ende des Kabels sind um mehrals eine Wellenlänge in der Phase gegeneinander verschoben. Da die Phasenverschiebung um ganze Wellenlängen in den Konstanten nicht zum Ausdruck kommt, so zicht man zweckmäßig von vornherein $2 \pi$ von dem Werte von $\alpha 1$ ab und schreibt

$$
\gamma 1=0,423+i(2 \pi+0,834) \text {. }
$$

Die Konstanten $\mathfrak{U}, \mathfrak{B}, \mathfrak{C}$ (die Kennstriche seien vorläufig weggelassen) ergeben sich aus der Charakteristik und der Fortpflanzungskonstanten folgendermaßen:

$$
\mathscr{A}=\frac{\mathrm{e}^{\gamma \mathrm{I}}+\mathrm{e}^{-\gamma 1}}{2}=\operatorname{\operatorname {coj}} \gamma 1 ; \mathfrak{B}=3 \cdot \frac{\mathrm{e}^{\gamma 1}-\mathrm{e}^{-\gamma \mathrm{l}}}{2}=3 \cdot \sin \gamma 1 ; \tilde{C}=\frac{\operatorname{Sin} \gamma \mathrm{l}}{8} \text {. }
$$

Die Berechnung erfolgt am einfachsten unter Benutzung von Funktionentafeln, z. B. Smithsonian Mathematical Tables, Hyperbolic Functions ${ }^{\mathbf{1}}$ ).

1) Verlag Smithsonian Institution, City of Washington. Die Tafeln enthaiten fünfstellige Logarithmen der hyperbolischen Funktionen, die natürlichen hyperbolischen Zahlen fünfstellig, fünfstellige Werte der Kreisfunktionen sinus und cosinus sowie ihre Logarithmen, gleichfalls fünfstellig, und die zugehörigen Winkel und Bogen, ferner Exponentialfunktionen $n, \log _{10}\left(\mathrm{e}^{\mathrm{n}}\right)$, $\mathrm{e}^{\mathrm{n}}$, e-n, die natürlichen Logarithmen der Zahlen von 0-Io ooo, sowie die Gudermannschen Winkel (Hyperbelamplituden). 
Es ist also

$\left.\mathfrak{A}=\mathfrak{C o j} \gamma l=\mathfrak{C o j} \beta l \cos \alpha 1+i \operatorname{Sin} \beta l \sin \alpha 1^{1}\right)$. Aus den Smithsonian Tables ist zu ent-

$$
\begin{aligned}
& \left.=\mathrm{I}, \mathrm{Og} \mathrm{I} \cdot 0,672+\mathrm{i} 0,436 \cdot 0,74 \mathrm{I}^{2}\right) \\
& =0,735+\mathrm{i} 0,323=0,803 \cdot \epsilon^{\mathrm{i} 23,70} .
\end{aligned}
$$

[nehmen:

Die Umrechnung der algebraischen Form der komplexen Größe in die geometrische Form erfolgt wohl am einfachsten und mit genügender Genauigkeit unter Benutzung der im Hilfsbuch für die Elektrotechnik von K. Strecker auf S. 9I angegebenen Tafel ${ }^{3}$ ) und mit Hilfe des Rechenschiebers, und zwar folgendermaßen:

$$
\begin{aligned}
\frac{0,323}{0,735}=0,439= & \operatorname{tg} \alpha \text { (vgl. Fig. 2). Aus der Tabelle zu entnehmen: } \\
\alpha & =23,7^{0} ; \frac{\mathrm{I}}{\cos \alpha}=\mathrm{I}, \mathrm{C} 93 \\
\mathrm{c} & =\mathrm{a} \cdot \frac{\mathrm{I}}{\cos \alpha} \cdot \mathrm{e}^{\mathrm{i} 23,7^{\circ}}=0,803 \cdot \mathrm{e}^{\mathrm{i} 23,7^{\circ}}=\mathfrak{H}^{\prime} .
\end{aligned}
$$

Für mit dem Rechnen mit komplexen Zahlen nicht Geübte empfiehlt es sich, stets den Vektor und die beiden Ordinaten zu

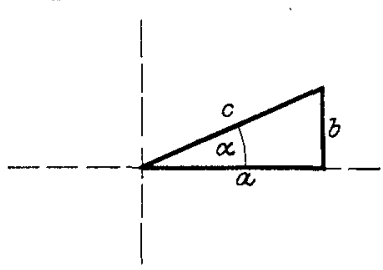

Fig. 2. zeichnen. Auf diese Weise lassen sich am sichersten Fehler vermeiden. Bei meinen vielen Rechnungen hat es sich mir als zweckmäßig erwiesen, die Zahlen folgendermaßen hinzuschreiben:

$$
\begin{array}{r}
\left.0,735+\mathrm{i} 0,323 \underset{(\operatorname{tg} \alpha)}{0,439} \underset{(\alpha)}{23,7^{0}} \mathrm{I}, \operatorname{cg} 3\right] \\
\left(\frac{1}{\cos \alpha}\right)
\end{array}
$$

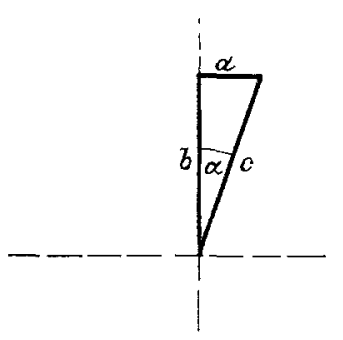

Fig. 3.

$\operatorname{Sin} \gamma 1=\operatorname{Sin} \beta 1 \cdot \cos \alpha 1+i \operatorname{Co}_{0 j} \beta 1 \cdot \sin \alpha 1$

$$
\begin{aligned}
& =0,436 \cdot 0,672+\mathrm{i} I, 09 \mathrm{I} \cdot 0,74 \mathrm{I}=0,293+\text { i o,8 } \mathrm{ro} \text { (vgl. Fig. 3). } \\
& \frac{\mathrm{a}}{\mathrm{b}}=0,362 \quad \alpha=\mathrm{I} 9,9^{0} \quad \frac{\mathrm{I}}{\cos \alpha}=\mathrm{I}, 064 \\
& c=I, 064 \cdot 0,8 \mathrm{IO} \cdot \mathrm{e}^{\mathrm{i}\left(\frac{\pi}{2}-19,9^{\circ}\right)}=0,863 \cdot \mathrm{e}^{\mathrm{i} 70,1^{\circ}} \\
& \underline{B^{\prime}}=3 \cdot \operatorname{Sin} \gamma 1=278,5 \cdot \mathrm{e}^{-\mathrm{i} 2,3^{\circ}} \cdot 0,863 \cdot \mathrm{e}^{\mathrm{i} 70,1^{\circ}}=240 \cdot \mathrm{e}^{\mathrm{i} 67,8^{\mathrm{e}}} \\
& \underline{\mathbb{S}^{\prime}}=\frac{\mathrm{I}}{8} \cdot \sin \gamma 1=\underline{0,003 \mathrm{IO} \cdot \mathrm{e}^{\mathrm{i} 72,6^{\circ}} .}
\end{aligned}
$$

2. $500 \mathrm{~km}$ Pupinleitung.

Die Pupinleitung bestehe aus 4,5 mm starkem Bronzedraht; in Abständen von Io $\mathrm{m}$ seien Spulen mit einer Induktivität von 0,2 Henry und einem wirksamen Widertsand von 9 Ohm eingeschaltet. Eine solche Leitung hat folgende Eigenschaften:

Wirksamer Widerstand der Leitung für I km . . . . 2,5 $\mathrm{Ohm}$

Wirksamer Widerstand der Spulen für I km . . . . 0,9 ,

$$
\text { Zusammen } 3,4 \quad \mathrm{Ohm}=\mathrm{R}
$$

Induktivität der Leitung für $\mathrm{I} \mathrm{km}$. . . . . . . . . 0,002 Henry

Induktivität der Spulen für $\mathrm{rkm}$. . . . . . . . 0,020 ",

$$
\text { Zusammen } 0,022 \text { Henry }=\mathrm{L}
$$

1) Vgl. z. B. Funktionentafeln von Jahnke und Emde (Verlag von B. G. Teubner) S. II.

2) Größere Genauigkeit ist nicht erforderlich und, wenn man den Rechenschieber benutzen will, nicht möglich.

3) Vgl. auch Funktionentafeln von Jahnke und Emde, S. 5 . 
Kapazität der Leitung und der Spulen für I km . . . 0,0062 Mikrofarad $=\mathrm{C}$

Ableitung der Leitung und der Spulen für I km . . . $0,5 \quad$ Mikrosiemens $=G$

In einer solchen Leitung beträgt angenähert die spezifische Dämpfung:

$$
\beta=\frac{R}{2} \sqrt{\frac{C}{L}}+\frac{G}{2} \sqrt{\frac{L}{C}} .
$$

Mit dem Rechenschieber gerechnet ergibt sich

$$
\begin{aligned}
& \beta=\frac{\mathrm{I}, 7}{\mathrm{I} 885}+0,25 \cdot \mathrm{I} 885 \cdot \mathrm{IO}^{-6}=0,000902+0,00047 \mathrm{I}=0,001373 \\
& \alpha=\omega \sqrt{\mathrm{C} \cdot \mathrm{L}}=5000 \cdot \sqrt{0,0062 \cdot 0,022} \cdot \mathrm{IO}^{-3}=0,0584 \\
& Z=\sqrt{\frac{\mathrm{L}}{\mathrm{C}}}=\mathrm{I} 885 .
\end{aligned}
$$

Berechnet man die Werte nicht nach den Annäherungsformeln, sondern unter Verwendung siebenstelliger Logarithmen ohne Vernachlässigungen, so ergibt sich

$$
\begin{aligned}
& \beta=0,00139358 \\
& \alpha=0,059019 \\
& \beta=1943 \cdot \mathrm{e}^{-\mathrm{i} 4,93^{\circ}}
\end{aligned}
$$

Für die vorliegenden Berechnungen würde dieVerwendung der mit dem Rechenschieber nach den Annäherungsformeln gewonnenen Werte vollkommen genügen. Da die genaueren Werte aber vorliegen, soll im folgenden mit diesen weiter gerechnet werden.

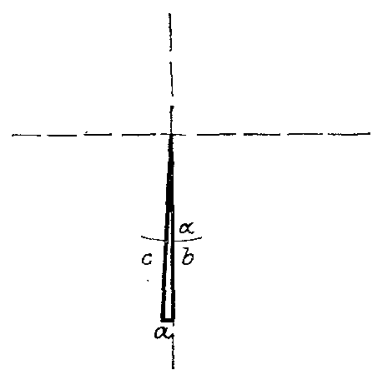

Fig. 4 .

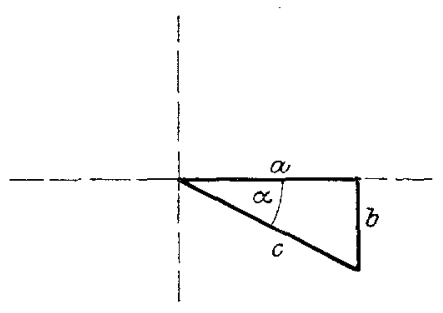

Fig. 5.

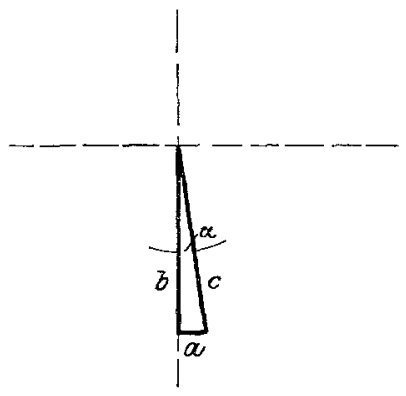

Fig. 6 .

Die Konstanten der Pupinleitung berechnen sich aus $\beta, \alpha$ und 8 in der gleichen Weise wie bei dem Kabel. Die Rechnung braucht also hier nicht durchgeführt zu werden.

Es ergibt sich

$$
\begin{aligned}
& \frac{\mathfrak{U}^{\prime \prime}=0,808}{\mathfrak{B}^{\prime \prime}=2370} \cdot \mathrm{e}^{\mathrm{i} 243,5^{\circ}} \\
& \frac{\mathrm{e}^{\mathrm{i} 254,9^{\circ}}}{\mathfrak{S}^{\prime \prime}=0,000628} \cdot \mathrm{e}^{\mathrm{i} 264,8^{\circ}}
\end{aligned}
$$

3. Kabel und $500 \mathrm{~km}$ Pupinleitung.

Nunmehr können die Konstanten des ganzen Systems berechnet werden, und zwar mit dem Rechenschieber unter Benutzung der Tabelle des Hilfsbuches. Es ist

$$
\begin{aligned}
\mathfrak{U}_{1} & =\mathfrak{U}^{\prime} \mathfrak{U}^{\prime \prime}+\mathfrak{B}^{\prime} \mathfrak{C}^{\prime \prime} . \\
\mathfrak{U}^{\prime} \mathfrak{U}^{\prime \prime} & =0,649 \cdot \epsilon^{\mathrm{i} 267,3^{\circ}} \text { (vgl. Fig. 4). }
\end{aligned}
$$

DerVektor liegt im dritten Quadranten. a sowohl wie b haben ein negatives Vorzeichen. $\alpha$ ist gleich $2,7^{0}$. Aus der Tabelle entnehmen wir $\operatorname{tg} \alpha=0,0475$ und $\frac{I}{\cos \alpha}=I$,oor, und zwar 
zweckmäßig in dieser Reihenfolge; es ergibt sich nun $b=\frac{c}{I / \cos \alpha}=0,649: I, 00 I=0,647$ und $\mathrm{a}=\mathrm{b} \cdot \operatorname{tg} \alpha=0,647 \cdot 0,0475=0,03 \mathrm{I}$, also

$$
\begin{aligned}
& \mathfrak{U}^{\prime} \mathfrak{U}^{\prime \prime}=-0,03 \mathrm{I}-\mathrm{i} 0,647 \\
& \mathfrak{B}^{\prime} \mathfrak{C}^{\prime \prime}=0,1508^{\circ} \cdot \mathrm{e}^{\mathrm{i} 332,6^{\circ}} \text { (vgl. Fig. 5). }
\end{aligned}
$$

Der Vektor liegt im vierten Quadranten. $\alpha$ ist gleich $27,4^{\circ}$. a hat ein positives Vorzeichen, $\mathrm{b}$ ein negatives. Es ergibt sich in ganz der gleichen Weise wie vorher

$$
\mathfrak{B}^{\prime} \mathfrak{C}^{\prime \prime}=+0, \mathrm{r} 34-\mathrm{i} 0,069 \text {. }
$$

Nun kann man $\mathfrak{A}^{\prime} \mathfrak{H}^{\prime \prime}$ und $\mathfrak{B}^{\prime} \mathfrak{C}^{\prime \prime}$ addieren. Es ergibt sich

$$
\mathfrak{U}^{\prime} \mathfrak{A}^{\prime \prime}+\mathfrak{B}^{\prime} \mathfrak{C}^{\prime \prime}=+0, \text { I03 }- \text { i } 0,7 \text { I6 }=\mathfrak{A}_{1} \text {. }
$$

In der weiteren Rechnung braucht man $\mathfrak{A}_{1}$ in der geometrischen Form. Diese findet man folgendermaßen: Der Vektor liegt im vierten Quadranten, wie in der Fig. 6 angegeben, da a positiv und $\mathrm{b}$ negativ ist. $\operatorname{tg} \alpha$ ist gleich $0,103 / 0,7 \mathrm{I} 6=0, \mathrm{I}_{44}$.

Aus der Tabelle entnimmt man nun $\alpha=8,2^{0} ; \frac{I}{\cos \alpha}=I$,oIo; also

$$
\mathrm{c}=0,7 \mathrm{I} 6 \cdot \mathrm{I}, \mathrm{OI} \cdot \mathrm{e}^{\mathrm{i}(270+8,2)^{0}}=0,724 \cdot \mathrm{e}^{\mathrm{i} 278,2^{\circ}}
$$
Reihen:

Man schreibt zweckmäßig die Rechnung übersichtlich in folgender Form in drei $\mathfrak{A}^{\prime} \mathfrak{U}^{\prime \prime}=0,649 \cdot \mathrm{e}^{\mathrm{i} 267,3^{\circ}} \quad\left[2,7^{0} 0,0475\right.$ I,00I $]=-0,03 \mathrm{I}-\mathrm{i} 0,647$ $\mathfrak{B}^{\prime}\left[\mathbb{C}^{\prime \prime}=0,1508 \cdot \mathrm{e}^{\mathrm{i} 332,6^{\circ}}\left[27,4^{0} 0,5 \mathrm{I9}\right.\right.$ I,I27 $]=+0,134-\mathrm{i} 0,069$

$$
\begin{aligned}
& =+0, \mathrm{I03}-\mathrm{i} 0,7 \mathrm{I} 6\left[0, \mathrm{I} 4448,2^{0} \quad \text { I,OIO }\right] \\
& =0,724 \cdot e^{\mathrm{i} 278,2^{0}}=\mathfrak{A}_{1}
\end{aligned}
$$

In ähnlicher Weise ergeben sich

$$
\mathfrak{U}_{2}=7,73 \cdot \mathrm{e}^{\mathrm{i} 323,1^{\circ}} \quad \mathfrak{B}=2070 \cdot \mathrm{e}^{\mathrm{i} 281,7^{\circ}} \quad \subseteq=0,002955 \cdot \mathrm{e}^{\mathrm{i} 311,5^{\circ}}
$$

Wenn man die Konstanten berechnet hat, macht man zweckmäßig zunächst die Probe, ob man keine Rechenfehler gemacht hat. Die Konstanten müssen nämlich der Bedingung genügen: $\mathfrak{A}_{1} \mathfrak{A}_{2}-\mathfrak{B} \mathfrak{C}=\mathrm{I}$. Es ergibt sich

$$
\begin{aligned}
& \mathfrak{A}_{1} \mathfrak{A}_{2}=5,59 \cdot \mathrm{e}^{\mathrm{i} 241,3^{0}}\left[28,7^{0} \quad 0,547 \text { I, I } 40\right]=-2,68-\mathrm{i}_{4}, 90 \\
& \mathfrak{B C}=6, \mathrm{II} \cdot \mathrm{e}^{\mathrm{i} 233,2^{\circ}}\left[36,8^{\circ} 0,747 \mathrm{I}, 24^{8}\right]=-3,67-\mathrm{i} 4,9^{\circ} \\
& \mathfrak{A}_{1} \mathfrak{A}_{2}-\overline{\mathfrak{B} \mathbb{C}}=+0,99-\mathrm{i} 0,00
\end{aligned}
$$

Die Genauigkeit genügt für den vorliegenden $Z$ weck durchaus.

Nunmehr kann die Fortpflanzungskonstante $\gamma 1$ berechnet werden.

$$
\gamma 1=\ln \left(\sqrt{\mathfrak{A}_{1} \mathfrak{A}_{2}}+\sqrt{\sqrt[\mathfrak{A}_{1} \mathfrak{A}_{2}]{-I}}\right)^{1}=\ln \left(\sqrt{\mathfrak{A}_{1}} \overline{\mathfrak{A}_{2}}+\sqrt{\mathfrak{B} \mathfrak{C}}\right)
$$

Es ergibt sich

$$
\sqrt{\mathscr{\mathscr { A }}_{1} \mathfrak{\mathfrak { A }}_{2}}+\sqrt{\sqrt{\mathfrak{B} \mathfrak{S}}}=-2,3 \mathrm{II}+\mathrm{i}_{4,252}=4,84 \cdot \mathrm{e}^{\mathrm{i}(118,5+\mathrm{x} \cdot 2 \pi)^{0}} .
$$

Das $\mathrm{x}$ ist aus der Überlegung darüber zu entnehmen, um wieviel Wellenlängen auf den einzelnen Leitungen die Ströme am Anfang und Ende gegeneinander in der Phase verschoben sind. Es ist schon am Anfang gesagt worden, daß die Ströme am Anfang und am Ende des Kabels um mehr als eine Wellenlänge in der Phase gegeneinander verschoben sind. Das $\alpha 1$ betrug $2 \pi+0,834$. Für die Pupinleitung beträgt die Wellenlängenkonstante $\alpha 1=9 \pi+\mathrm{r}, 28, \mathrm{~d}$. h. die Ströme sind um mehr als $4 \frac{1}{2}$ volle Wellenlänge in der Phase verschoben. Über das ganze System sind sie also sicher um mehr als $5 \frac{1 / 2}{2}$ volle Wellenlängen verschoben. Man wird also $\mathrm{x}=6$ setzen müssen.

1) Vgl. Breisig, Theoretische Telegraphie, S. 315 . 
Es ergibt sich

$$
\gamma \mathrm{l}=\ln 4,8,4 \cdot \mathrm{e}^{\mathrm{i} 2278,5^{\circ}}=\mathrm{I}, 573+\mathrm{i} 39,7=\beta \mathrm{l}+\mathrm{i} \alpha .1
$$

Da der Dämpfungsexponent des Kabels 0,423, der der Pupinleitung 0,697, die algebraische Summe demnach I,I20 beträgt, so beläuft sich der durch Reflexionen hervorgerufene Zuwachs des Dämpfungsexponenten auf I,573 - I,I20 = 0,453. Der Zuwachs durch Reflexionen ist also größer als der Dämpfungsexponent des Kabels für sich.

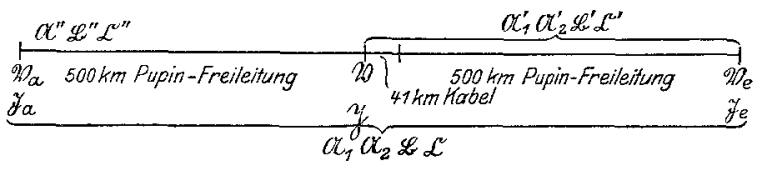

Fig. 7 .

b) $43 \mathrm{~km}$ Krarupkabel zwischen zweimal $500 \mathrm{~km}$ Pupinleitung (vgl. Fig. 7).

Es seien $\mathfrak{A}^{\prime \prime} \mathfrak{B}^{\prime \prime} \mathbb{C}^{\prime \prime}$ wie vorher die Konstanten der $500 \mathrm{~km}$ langen Pupinleitung, $\mathfrak{A}_{1}^{\prime}$ $\mathfrak{A}_{2}^{\prime} \mathfrak{B}^{\prime} \mathfrak{C}^{\prime}$ die eben gefundenen Konstanten des Leitungssystems aus Kabel und Pupinleitung, $\mathfrak{A}_{1} \mathfrak{U}_{2} \mathfrak{B} \mathbb{C}$ die Konstanten des Ganzen. Es bestehen dann die Beziehungen:

$$
\begin{aligned}
& \text { x. } \mathfrak{B}_{\mathrm{a}}=\mathfrak{Q}^{\prime \prime} \mathfrak{\mathfrak { F }}+\mathfrak{B}^{\prime \prime} \mathfrak{S} ; \quad \mathfrak{S}_{\mathrm{a}}=\mathfrak{U}^{\prime \prime} \mathfrak{\mathfrak { S }}+\mathfrak{C}^{\prime \prime} \mathfrak{B} \\
& \text { 2. } \mathfrak{B}=\mathfrak{U}_{1}^{\prime} \mathfrak{B}_{\mathrm{e}}+\mathfrak{W}^{\prime} \mathfrak{J}_{\mathrm{e}} ; \quad \widetilde{\mathfrak{J}}=\mathfrak{U}_{2}^{\prime} \mathfrak{W}_{\mathrm{e}}+\mathfrak{C}^{\prime} \mathfrak{S}_{\mathrm{e}} \\
& \text { 3. } \mathfrak{V}_{\mathrm{a}}=\mathfrak{A}_{1} \mathfrak{B}_{\mathrm{e}}+\mathfrak{B} \mathfrak{\mathfrak { F }}_{\mathrm{e}} ; \quad \mathfrak{\Im}_{\mathrm{a}}=\mathfrak{A}_{2} \mathfrak{\mathfrak { V }}_{\mathrm{e}}+\mathfrak{C} \mathfrak{V}_{\mathrm{e}}
\end{aligned}
$$

Durch Elimination von $\mathfrak{B}$ und $\mathfrak{J}$ aus $I$. und 2. ergibt sich

$$
\begin{array}{ll}
\mathfrak{A}_{1}=\mathfrak{A}^{\prime \prime} \mathfrak{U}_{1}^{\prime}+\mathfrak{B}^{\prime \prime} \mathfrak{C}^{\prime} & \mathfrak{A}_{2}=\mathfrak{A}^{\prime \prime} \mathfrak{A}_{2}^{\prime}+\mathfrak{C}^{\prime \prime} \mathfrak{B}^{\prime} \\
\mathfrak{B}=\mathfrak{U}^{\prime \prime} \mathfrak{B}^{\prime}=\mathfrak{B}^{\prime \prime} \mathfrak{U}_{2}^{\prime} & \mathfrak{C}=\mathfrak{U}^{\prime \prime} \mathfrak{C}^{\prime}+\mathfrak{C}^{\prime \prime} \mathfrak{A}_{1}^{\prime}
\end{array}
$$

Die Ausführung der Rechnungen unterscheidet sich nicht von der unter a). Es ergibt sich:

$$
\begin{array}{ll}
\mathfrak{U}_{1}=7,46 \cdot \mathrm{e}^{\mathrm{i} 203,1^{0}} & \mathfrak{U}_{2}=7,46 \cdot \mathrm{e}^{\mathrm{i} 203,1^{0}} \\
\mathfrak{B}=\mathrm{Ig} 380 \cdot \mathrm{e}^{\mathrm{i} 214,0^{\circ}} & \mathfrak{C}^{\mathfrak{C}}=0,0028 \mathrm{I} \cdot \mathrm{e}^{\mathrm{i} 193,1^{0}}
\end{array}
$$

Da die Leitung nunmehr wieder symmetrisch ist, ergibt sich für $\mathfrak{U}_{1}$ und $\mathfrak{H}_{2}$ der gleiche Wert. Der Dämpfungsexponent beträgt für das Ganze 2,700, während die einzelnen Teile nur einen Beitrag von zusammen I,8 6 liefern. Der Zuwachs durch Reflexionen beträgt also 0,884 .

Es bleibt noch ein Wort zu sagen über die Charakteristik der zusammengesetzten Leitungen. Bei unsymmetrischen Leitungen ist sie bekanntlich an den beiden Enden gemessen eine verschiedene. Es beträgt

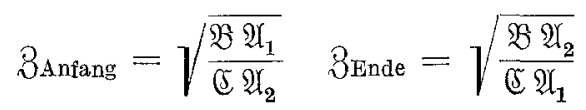

Für den Fall a des Kabels + 500 km Pupinleitung ergibt sich danach für die Kabelseite eine Charakteristik

und für die andere Seite

$$
3 \mathrm{Kabel}=256 \cdot \mathrm{e}^{-\mathrm{i} 37,7}
$$

$$
8 \text { Freileitung }=2740 \cdot \mathrm{e}^{+\mathrm{i} 7,5^{\circ}}
$$

Für den Fall b) der Einschaltung des Kabels zwischen zwei Pupinleitungen von je $500 \mathrm{~km}$ Länge ergibt sich eine Charakteristik von

$$
B=2628 \cdot \mathrm{e}^{\mathrm{i} 10,4^{\circ}}
$$

Kommt es nur darauf an, den Wert des Dämpfungsexponenten festzustellen, so kann man bei größeren Werten, etwa von $2,5 \mathrm{ab}$, den Dämpfungsexponenten auch angenähert setzen 
I. bei symmetrischen Leitungen

$$
\beta 1=\ln 2 \mathfrak{A},
$$

2. bei unsymmetrischen Leitungen

$$
\beta 1=\ln 2 \sqrt{\mathfrak{Q}_{1} \mathfrak{H}_{2}} .
$$

Im allgemeinen wird man aber, schon um die Probe auf die Richtigkeit der Rechnung machen zu können, $\mathfrak{S}$ und $\mathfrak{C}$ auch berechnen.

4. Dämpfungszuwachs durch Reflexionen für bestimmte Fälle. Auf gleiche Weise wie im vorigen Abschnitt ist noch für mehrere Fälle der Dämpfungszuwachs berechnet worden. Die Werte sind in folgender Tabelle zusammengestellt:

$$
\begin{aligned}
& 43 \mathrm{~km} \text { Krarupkabel }\left(8=278,5 \cdot \mathrm{e}^{-\mathrm{i} 2,3^{0}}\right)+500 \mathrm{~km} \text { Pupinleitung } \\
& \left(8=\mathrm{I} 943 \cdot \mathrm{e}^{-\mathrm{i} 4,9^{\circ}}\right) \text {. . . . . . . . . . . . . . . . . . } 0,453 \\
& 43 \mathrm{~km} \text { Krarupkabel }\left(8=278,5 \cdot \mathrm{e}^{-\mathrm{i} 2,3^{0}}\right)+\text { rooo } \mathrm{km} \text { Pupinleitung } \\
& \left(8=\mathrm{r} 943 \cdot \mathrm{e}^{-\mathbf{i} 4,9^{\circ}}\right) \text {. . . . . . . . . . . . . . . . . . } 0,469 \\
& 500 \mathrm{~km} \text { Pupinleitung }+43 \mathrm{~km} \text { Krarupkabel }+500 \mathrm{~km} \text { Pupinleitung . 0,884 } \\
& \text { ro00 km Pupinleitung }+43 \mathrm{~km} \text { Krarupkabel }+1000 \mathrm{~km} \text { Pupinleitung } 0,870 \\
& 43 \mathrm{~km} \mathrm{Krarupkabel}+500 \mathrm{~km} \text { gewöhnliche Freileitung }\left(8=577 \cdot \mathrm{e}^{-\mathrm{i} 6,5^{\circ}}\right) \quad 0,080 \\
& 43 \mathrm{~km} \text { Pupinkabei }(8=800)+500 \mathrm{~km} \text { Pupinleitung }\left(8=\mathrm{I943} \cdot \mathrm{e}^{-\mathrm{i} 4,9^{0}}\right) \quad 0,100 \\
& 43 \mathrm{~km} \text { Pupinkabel }+500 \mathrm{~km} \text { gewöhnliche Freileitung . . . . . . 0,000 }
\end{aligned}
$$

Danach sind die Reflexionsverluste weder für Pupinkabel noch für Krarupkabel erheblich, wenn sie an gewöhnliche Freileitungen angeschlossen werden. Bei Anschaltung von Krarupkabeln so niedriger Charakteristik, wie sie starkdrähtige Unterwasserkabel haben, an Pupinleitungen hoher Charakteristik sind die Reflexionswerte sehr groß. Auch bei dem Pupinkabel nach dem Muster des englisch-belgischen Kabels treten beim Anschalten an Pupinleitungen hoher Charakteristik noch geringe Reflexionsverluste auf. Es ist aber zu bemerken, daß das Kabel aus besonderer Vorsicht sehr schwach pupinisiert ist, da man den Eigenschaften der Guttapercha doch noch etwas mißtrauisch gegenüberstand. Es hätte unbedenklich noch eine etwas höhere Charakteristik erhalten können, so daß die Reflexionsverluste bei Anschluß an Pupinfreileitungen vollständig wegfallen würden.

\section{Zusammenfassung.}

I. Papier-Luftraumkabel mit Bleimantel haben geringere Kapazität und geringere Ableitung, daher bei gleichen Abmessungen geringere Dämpfung als Guttaperchakabel; sie haben aber geringere mechanische Festigkeit und sind bei gleicher Leistungsfähigkeit schwerer als Guttaperchakabel. Sie sind also in elektrischer Beziehung den Guttaperchakabeln überlegen, in mechanischer Beziehung sind sie ihnen jedoch nicht gleichwertig.

Papier-Luftraumkabel können daher für Unterseeverbindungen nur an Stellen verwendet werden, wo sie nicht durch Strönungen, schleifende Anker usw. gefährdet sind. Auch kommen sie ihrer Schwere wegen nur an flachen Stellen in Betracht.

2. Wenn zur Verringerung der Dämpfung die Induktivität der Kabel erhöht werden soll, so geschieht dies besser durch Einschaltung von Spulen nach Pupin als durch Umwickelung der Leiter mit Eisendraht nach Krarup. Durch die Pupinisierung wird die gleiche Güte der Sprechverständigung billiger erreicht und dabei gleichzeitig den Kabeln eine günstigere Charakteristik erteilt. Die Kabel mit eisenumsponnenem Leiter sind ihrer niedrigen Charakteristik wegen nur für Anschaltung an gewöhnliche Freileitungen geeignet; bei der Anschaltung an Pupin-Freileitungen entstehen erhebliche Verluste durch Reflexionen. Pupinkabeln kann man dagegen eine Charakteristik geben, die sie zur: Anschaltung an gewöhnliche Freileitungen und an Pupin-Freileitungen gleich geeignet machen. 